\title{
DEVELOPMENT OF A PETROLEUM PIPELINE MONITORING SYSTEM FOR CHARACTERIZATION OF DAMAGES USING A FOURIER TRANSFORM
}

\author{
E. N. Aba' ${ }^{1, *}$, O. A. Olugboji2 ${ }^{2}$ A. Nasir ${ }^{3}$, M. A. Olutoye ${ }^{4}$ and O. Adedipe ${ }^{5}$ \\ $\mathbf{1 , 2 , 3 , 5}$ Dept. of MeChanical Engineering, Federal Univ. Of TeChnology, MinNa, Niger State, NIGERIA \\ 4,Department of Chemical Engineering, Federal Univ. Of TeChnology, MinNa, Niger State, Nigeria \\ E-mail addresses: ${ }^{1}$ emmaaba22@yahoo.com; ${ }^{2}$ olubojioluwafemi@futminna.edu.ng; \\ 3 a.nasir@futminna.edu.ng; ${ }^{4}$ m.olutoye@futminna.edu.ng; ${ }^{5}$ oye.adedipe@futminna.edu.ng
}

\begin{abstract}
Significant damage to the environment and huge economic losses are potential problems caused by leakage from petroleum pipelines. The occurrence of a leakage in a pipeline throughout its lifetime is very difficult to prevent. To minimize environmental damage and high economic losses, an efficient pipeline monitoring system is required to carry out damage characterization thereby enhancing quick response. The signal processing technique of sampling and reconstruction was adopted and mathematical algorithms for the characterization of damages in pipes were developed using the Fourier transform method. These were simulated with the results showing a good agreement between the shapes and magnitudes of the measured original and reconstructed pulses. The simulation was verified with experiments on the test rig. The results showed an underestimation in the magnitudes of the reconstructed pulses in the range of $40-45 \%$. This problem was solved by using a factor $K$ obtained by dividing the maximum amplitude value of the original pressure pulse by that of the reconstructed pulse. Reconstruction of the measured original pulse at a damage location was achieved from combining the measured pulses from two other close locations using the developed Fourier transform based model.
\end{abstract}

Keywords: Damage Pipeline-monitoring Characterization Fourier transform Reconstruction

\section{INTRODUCTION}

Major pipelines across the world transport large quantities of crude oil, natural gas, and petroleum products. These pipelines play an important role in modern societies and are crucial in providing needed fuels for sustaining vital functions such as power generation, heating supply, and transportation. In light of the hazardous properties of the products being transmitted through these pipelines, a ruptured pipeline has the potential to do serious environmental damage. Ruptured pipelines also cause huge economic as well as humanitarian losses. The risk associated with pipeline in terms of safety of people, damage to the environment and loss of income has been a major concern to pipeline integrity managers. Factors, such as external impacts, environmental corrosion, internal erosion, material initial defects, ground surface movements, improper maintenance and human reasons (vandalization, etc) may lead to pipeline leakage.

Pipeline inspection technologies using sensor networks have drawn significant attention, for example, in the applications of natural gas pipeline inspection and monitoring by acoustic sensors [1, 2]. This study intends to use sensor networks to characterize and classify damages in pipeline systems using pressure guided waves or pulses, and a signal processing techniques based on the principle of vibration in pipes. In general, pipeline defects can occur in the manufacture, construction, and operation processes.

${ }^{*}$ Corresponding author, tel: $+234-806-710-4205$ 
In this study, focus is on the operational defects that encompass fatigue, third party damage, denting and buckling. To ensure the continued safe operation of the transmission pipelines, continuous monitoring or periodic assessment of the integrity of the pipelines is necessary. In pipeline monitoring and inspection, the ultimate objective is to identify the locations that have defects, and obtain an accurate measurement and assessment of the defects so that human operators can take appropriate action to prevent further damage to the pipelines and environment. The goal of this study is to develop a continuous, remote, and realtime monitoring and inspection system using wire/wireless sensors that can characterize various impulsive events that cause damage to pipelines.

A pipeline monitoring and inspection system has a long list of tasks to accomplish ranging from detection of damage to determining structural defects in pipes. To achieve these goals, various actuators and sensors are relied upon. There are many types of sensors that have been studied and tested for pipeline inspections including acoustic sensors, fibre-optic sensors, and magnetostrictive sensors. Each type of sensors has its unique feature and operational condition. In this study, piezoelectric sensors were used

Vibration-based approaches have been developed in the past and have been proven to be relatively successful in detecting damage in pipes [3]. For a long period of development, vibration-based and guided waves-based damage detection technologies have produced a good number of research results. Olugboji [4] developed and tested mathematical techniques for locating an impulsive event on a pipeline using the pressure pulse caused by it from measurements made remotely. His experimental work was carried out using an experimental test rig comprising a flexible hose pipe with four pressure sensors distributed along the pipe and connected to a data acquisition system. The experiments were tested using static air in the pipe, and were found to give good results.

Junxiao et al. [5] carried out damage detection in a gas pipeline making use of the negative pressure wave (NPW), a stress wave generated by leakage in the pipeline which propagates along the pipeline from the leakage point to both ends, and the hoop strain variation along the pipe the leakage point to both ends, and the hoop strain variation along the pipe wall. Guofeng et al. [6], developed a wavelet packet-based damage index matrix to identify the crack damage in pipeline structure using a stress wave propagation approach with piezo-ceramic transducers. In their work, four cracks were artificially cut on the specimen, and each crack had six damage cases corresponding to different crack depths. This aided them to simulate cracks at different locations with different damage degrees. In each damage case, they used one piezoceramic transducer as an actuator to generate a stress wave to propagate along the pipeline specimen, and the other piezo-ceramic transducers were used as sensors to detect the wave responses. Golmohamadi [7] used wavelet transform for processing signals to recognize damage and leak location in a hardwarebased technique which used ultrasonic wave emission. Changhang et al. [8] used a low-power piezo-ceramic transducer as the actuator of vibrothermography and explored its ability to detect multiple surface cracks in a metal part. They employed the Fourier Transform signal processing technique in their work and their results showed that all cracks can be detected conveniently and simultaneously by using the proposed low-power vibrothermography. Enrique et al. [9] combined the guided waves and electromechanical impedance techniques based on smart sensing to define a new and integrated damage detection procedure. This combination of techniques was studied by them and they proposed a new integrated damage indicator based on ElectroMechanical Power Dissipation (EMPD). They tested the applicability of their proposed technique through different experimental tests, with both lab-scale and real-scale structures. Kia et al. [10] proposed a new approach to damage detection of a concrete column structure subjected to blast loads using embedded piezo-ceramic smart aggregates (SAs). They proposed active-sensing based approach in which the embedded SAs act as actuators and sensors that can respectively generate and detect stress waves.

Pipeline inspection technologies using sensor networks have drawn significant attention around the world. This study intends to use sensor networks to characterize bursts, leaks and other anomalies (damages) in general pipeline systems using pressure pulses and signal processing techniques based on the principle of vibration in pipes. The competitive advantage of this research work and its contribution to knowledge lies in its ability to perform real-time damage characterization through the use of a combination of wave propagation, an active sensor network, along with the digital signal processing technique of sampling and reconstruction; and also the ability of the developed system to carry out event reconstruction of the original pressure pulse at a 
particular sensor location by combining the pressure pulses from other sensor locations along the pipeline. This would make the interpretation of the cause of damage on pipes possible and would enhance faster response to damage by technicians. It would also assist the technicians in knowing what kind of repair work to do before arrival at the scene of the event and be armed with the requisite tools when signalled with a pipeline leak or rupture alarm. The early adopters for this technology would be petroleum companies; innovative pipeline maintenance companies, and government with obligation to maintain uninterrupted petroleum products supply.

\section{MATERIALS AND METHODS}

\subsection{Materials}

The major materials to be used for this research work are:

i. Flexible polyethylene hose pipe

ii. Water

iii. $\quad 75$ and 100 percent concentrated hydrochloric acid $(\mathrm{HCl})$

iv. Water tank

The major equipment for the research and a brief about their use is presented thus:

- Pressure sensors (piezoelectric sensors): For detection of propagated pulses along pipeline.

- Pulse generator: For generation of sharp fronted pressure pulses in the pipe.

- Pico Log 1000 Data acquisition module: Recording and processing of signals from pressure pulses at specified sampling rate

- Matlab ${ }^{\circledR}$ software package: Analysis of generated data; calculation of Fourier. functions of generated pulses; for sampling and reconstruction of signals

- Hand drill: To generated pulses in the pipe.

- Hammer: To generated pulses in the pipe.

- Laptop computer: Connected to data acquisition module for processing of and visualisation of data.

\subsection{Methods}

Pipelines are considered as one of the safest means of transporting petroleum products. These pipelines are damaged from time to time as a result of natural events (erosion, earthquakes, etc) or due to third party activities (explosions, drilling activities, vehicular movement, etc). A great challenge that pipeline operators have faced in the past even in situations where it had been known that damage has occurred along pipelines is the difficulty in pin-pointing the exact cause of the damage.

Most times, damages caused by impulsive events generate a pressure pulse that propagates in both directions through the fluid in the pipe. Detection and measurement of these pressure pulses can be carried out at points remote from the event. These measured pulses contain information about the event and can be used to characterize what must have caused the damage. The information about damage-causing impulsive event contained in these measured pressure pulses can be outputted through proper analysis of the pressure pulses (signals). Therefore, to achieve the characterization of damage events in pipes, proper signal analysis of measured pressure pulses is carried out.

\subsubsection{Numerical simulation and modeling of pressure pulse propagation for characterization} In this work, emphasis is based more on outputting, analyzing and categorizing the wave spectra obtainable from different impulsive or damage causing events. In terms of linking the characteristics of a signal to the 'physics' of the system that produces it, particularly with regard to the interactions of wave fields with matter and the design of sensors to record such interactions, the Fourier transform has and continues to rein supreme. This is because it provides the most general and comprehensive solution to physical systems described by differential equations and most of the laws of physics can be expressed in the form of differential equations [11]. The flowchart in Figure 1 illustrates the process involved in obtaining all the transforms derived in this work.

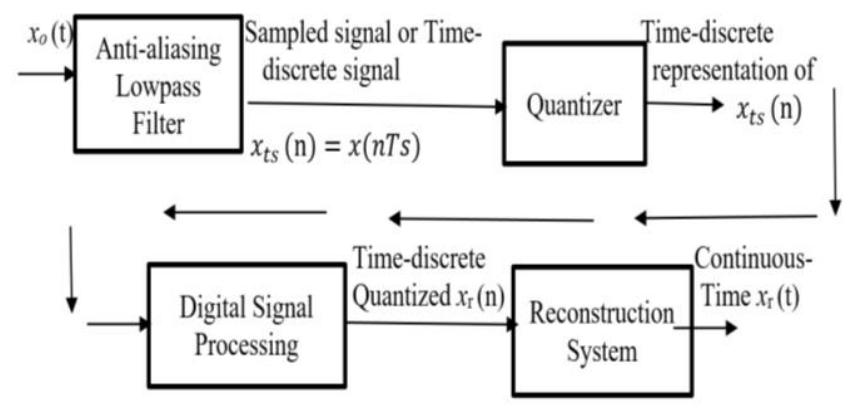

Figure 1: Flowchart of signal reconstruction process

\subsubsection{Mathematical Model for Sampling}

For this work, the Fourier Transform is employed as the basis to develop the mathematical model needed 
for the reconstruction of the signals arising from various impulsive events.

The following notations were adopted in the course of this work:

$x_{o}(\mathrm{t})=$ original analog signal

$x_{\mathrm{r}}(\mathrm{t})=$ reconstructed signal $\left(\right.$ where $\left.x_{\mathrm{r}}(\mathrm{t})=x_{o}(\mathrm{t})\right)$

$x_{t s}(n)=$ time-discrete signal or sampled signal

where

$f_{s}=$ sampling frequency (samples/second)

$T_{\mathrm{s}}=$ sampling interval (seconds/sample) (where $T_{s}=$

$\left.\frac{1}{f_{s}}\right)$

$\grave{\omega}=$ real angular frequency (radians/second)

$\omega=$ digital angular frequency (radians/second) (where

$\left.=\varphi T_{S}\right)$

$\mathrm{t}=$ continuous time variable

$\mathrm{n}=$ discrete integer variable

The inverse Fourier transform of a time discrete signal $x_{t s}(\mathrm{n})$ obtained from sampling $x_{0}(\mathrm{t})$ every $T s$ second is given by [12]:

$$
x_{t s}(n)=\frac{1}{2 \pi} \int_{-\pi}^{\pi} X_{t s}\left(e^{i w}\right) e^{i w n} d w
$$

In simplifying the above, the equation is expressed in terms of real angular frequency, $\varphi$. This gives:

$$
x_{t s}(n)=\frac{T_{s}}{2 \pi} \int_{-\frac{\pi}{T_{S}}}^{\frac{\pi}{T_{S}}} X_{t s}\left(e^{i \varphi T_{s}}\right) e^{i \varphi T_{s} n} d \varphi
$$

For a continuous signal, the inverse Fourier transform given by:

$$
X_{0}(t)=\frac{1}{2 \pi} \int_{-\infty}^{\infty} X_{o}(i \varphi) e^{i \varphi t} d \varphi
$$

Replacing $\mathrm{t}$ with $\mathrm{n} T \mathrm{~s}$ in the above equation, we obtain:

$$
X(n T s)=\frac{1}{2 \pi} \int_{-\infty}^{\infty} X_{o}(i \varphi) e^{i \varphi n T_{s}} d \varphi
$$

Splitting the integration in (4) into sub-intervals of length $\frac{2 \pi}{T_{s}}$ and taking the sum over the resulting integrals to obtain the complete area, we have:

$X(n T s)=\frac{1}{2 \pi} \sum_{k=-\infty}^{\infty} \int_{\frac{(2 k-1) \pi}{T_{S}}}^{\frac{(2 k+1) \pi}{T_{s}}} X_{o}(i \varphi) e^{i \varphi n T_{s}} d \varphi$

Changing the integration variable by setting $=\alpha+$ $\frac{2 * \pi k}{T_{S}}$, we obtain:

$$
\begin{aligned}
X(n T s)=\frac{1}{2 \pi} \sum_{k=-\infty}^{\infty} \int_{\frac{-\pi}{T_{S}}}^{\frac{\pi}{T_{S}}} X_{o}(i(\alpha \\
\left.\left.+\frac{2 * \pi k}{T_{s}}\right)\right) e^{i\left(\alpha+\frac{2 * \pi k}{T_{S}}\right) n T_{S}} d \alpha
\end{aligned}
$$

Multiplying (6) all through by $\frac{T_{S}}{T_{S}}$, with $\alpha=\varphi$ and noting that $e^{i 2 * \pi k n}$, we obtain:

$$
\begin{array}{r}
X(n T s)=\frac{T_{s}}{2 \pi} \int_{\frac{-\pi}{T_{s}}}^{\frac{\pi}{T_{S}}} \sum_{k=-\infty}^{\infty} \frac{1}{T_{s}} X_{o}(i(\varphi \\
\left.\left.+\frac{2 * \pi k}{T_{S}}\right)\right) e^{i \varphi n T_{s}} d \varphi
\end{array}
$$

In order for $x_{\text {ts }}(n)$ to be equal to $x(n T s)$ for all values of the integer $n$, Equations 2 and 7 must agree as given below:

$$
X_{t s}\left(e^{i \varphi T_{s}}\right)=\frac{1}{T_{s}} \sum_{k=-\infty}^{\infty} X\left(i\left(\varphi+\frac{2 \pi k}{T_{s}}\right)\right)
$$

The above is the mathematical model for sampling of signals to obtain their digital spectrum.

\subsubsection{Mathematical model for signal reconstruction}

The inverse Fourier transform for a bandlimited signal is given by [12]:

$$
x_{o}(t)=\frac{1}{2 \pi} \int_{-\frac{\pi}{T_{S}}}^{\frac{\pi}{T_{S}}} X(i \varphi) e^{i \varphi t} d \varphi
$$

$X_{t s}\left(e^{i \varphi T_{s}}\right)=\frac{X(I \varphi)}{T_{S}}$ for the interval being integrated and when this is substituted into equation 8 , we obtain:

$$
x_{o}(t)=\frac{T_{s}}{2 \pi} \int_{-\frac{\pi}{T_{S}}}^{\frac{\pi}{T_{S}}} X_{t s}\left(e^{i \varphi T_{S}}\right) e^{i \varphi t} d \varphi
$$

When the DTFT expression for $X_{t s}\left(e^{i \varphi T_{s}}\right)$ is applied, we obtain:

$$
x_{o}(t)=\frac{T_{S}}{2 \pi} \int_{-\frac{\pi}{T_{S}}}^{\frac{\pi}{T_{S}}} \sum_{k=-\infty}^{\infty} x_{t s}(n) e^{\left(-i \varphi n T_{S}\right)} e^{i \varphi t} d \varphi
$$

With summation and integration interchanged, we obtain:

$$
x_{o}(t)=\frac{T_{S}}{2 \pi} \sum_{k=-\infty}^{\infty} x_{t s}(n) \int_{-\frac{\pi}{T_{S}}}^{\frac{\pi}{T_{S}}} e^{i \varphi\left(t-n T_{S}\right)} d \varphi
$$

Integrating, we obtain:

$$
\begin{aligned}
& x_{r}(t)=x_{o}(t) \\
& =\sum_{k=-\infty}^{\infty} x_{t s}(n) \frac{\sin \left(\frac{\pi}{T_{s}}\left(t-n T_{s}\right)\right)}{\frac{\pi}{T_{s}}\left(t-n T_{s}\right)}
\end{aligned}
$$

The above is the reconstruction model that was used to recover the original signal $x_{o}(\mathrm{t})$ from the timediscrete sequence or sampled signal $x_{t s}(n)$.

To obtain a more accurate reconstruction of the original signal $x_{o}(\mathrm{t})$, we obtain:

$$
x_{r}(t)=\sum_{k=-\infty}^{\infty} x_{t s}(n) \frac{\sin \left(\frac{\pi}{T_{s}}\left(t-n T_{s}\right)\right)}{\frac{\pi}{T_{s}}\left(t-n T_{s}\right)} * K
$$


Where $\mathrm{K}$ is an approximation factor obtained by dividing the maximum value of $x_{o}(t)$ by the maximum value of $x_{r}(t)$.

\subsubsection{Simulation of mathematical model}

The simulation of these mathematical models developed for both the sampling and reconstruction of signals obtained from pressure pulses' propagating within a pipe was carried out with codes written using the MatLab software. This was done to ascertain the workability of the model and its ability to perform proper event reconstruction. The results of the simulation were good. These are shown in Figures 3, 4 , and 5.

\subsubsection{Experimentation for validation of simulation}

For the validation of the proposed models for damage location and characterization in pipes, three different experiments were carried out. These three experiments were carried out to mimic damage events in pipes caused by Explosion, Drilling and Vehicular movement respectively. The set-up consisted of a PVC (Polyvinyl Chloride) pipe of total length $25 \mathrm{~m}$ and internal diameter of $20 \mathrm{~mm}$ along which pressure pulses would propagate. Five sensors were located at different positions along the PVC pipe and connected to a single data instrumentation system to capture and record the propagation of the pulses. The set-up is shown in Figure 2.

To validate the theory of event location as discussed above; an experimental set up as shown in Figure 2 was built. This consisted of a water filled PVC (Polyvinyl Chloride) pipe of total length 20.11 metres and internal diameter of $20 \mathrm{~mm}$ along which pressure pulses propagated. A pressure pulse generator was be used to introduce sharp-fronted pulses into the water filled pipe. Six piezoelectric sensors were located at different positions along the PVC pipe. Sensor 1 was located at 2 metres from one end; sensor 2 was located at 5 metres from one end; sensor 3 was located at 8 metres from one end; sensor 4 was located at 11 metres from one end; sensor 5 was located at 14 metres from one end and sensor 6 was located at 17 metres from one end. Each sensor had a distance of 3 metres separating it from another sensor, and all sensors were connected to a single Pico Log data instrumentation system to capture and record the propagation of the pulses in the first experimental test rig. For the validation of the proposed methods for damage characterization in pipes, four different experiments were carried out. These four experiments were carried out to mimic damage events in pipes caused by explosion, drilling, vehicular movement, and corrosion respectively.

\subsubsection{Experimentation to mimic damage event caused by explosion}

A pulse generator was used to mimic an explosion in the pipe. Sensors connected to a single USB data logging were placed at known points along the pipe and an instrumentation system was used to capture the propagation of the pressure pulses and saved in a tab delimited data file. Analysis of the captured data was done offline using a program written in the MatLab programming language.

\subsubsection{Experimentation to mimic damage event caused by drilling}

The experimental setup was the same with that explosion. To mimic a drilling operation, sharp fronted pressure pulses were generated into the pvc pipe by using a hand drill to drill at different points along the surface of the pipe. The signals from the sensors were recorded using the data acquisition model and saved. The saved data was analysed using a program written in the Matlab ${ }^{\circledR}$ programming language.

\subsubsection{Experimentation to mimic a potential damage event caused by vehicular movement}

With a similar experimental setup as that of explosion and drilling, this experiment was carried out by using a hammer to strike at points along the pipe. The solid mass was meant to mimic a sudden weight like that obtained from a vehicle. The signals from the sensors were also recorded using the data acquisition device and saved. The saved data was then analysed using a program written in the Matlab $^{\circledR}$ programming language.

\subsubsection{Experimentation to mimic a potential dal age event caused by corrosion}

The experimental setup is as illustrated in Figure 2. To mimic a corrosive environment in a pipe, sharp fronted pressure pulses were generated into the water filled pvc pipe by carefully introducing concentrated hydrochloric acid at different points along the length of the pipe. The signals from the sensors were recorded using the data acquisition model and saved. The saved data was analysed using a program written in the Matlab ${ }^{\circledR}$ programming language. 


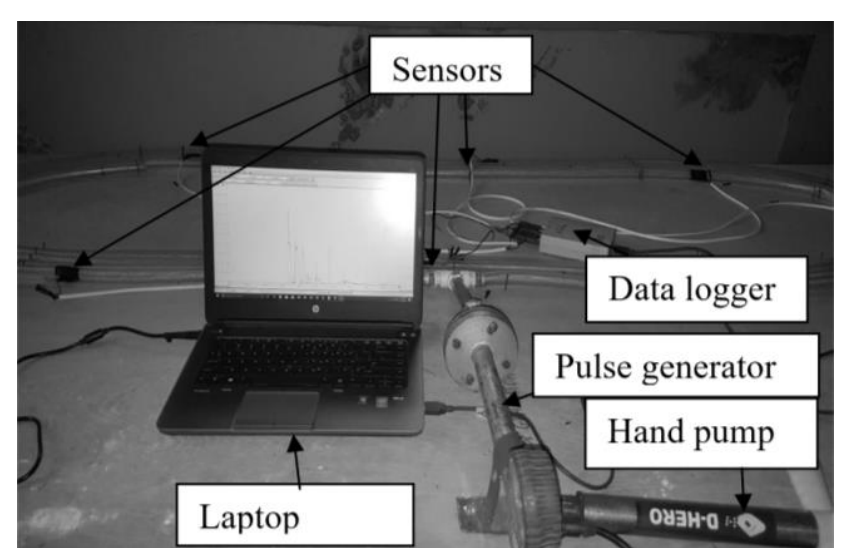

Figure 2: Experimental test rig showing various components of the rig

\section{RESULTS AND DISCUSSION}

\subsection{Simulation oŕ Derived Mathematical Models} Preliminary testing of the mathematical models for sampling ani reconstruction was carried out with the model being applied to three sample sets of data obtained from a different experimental setup carried out on pipeline monitoring. This simulation was carried out using the Matlab ${ }^{\circledR}$ software. The results obtained show that this model can exactly reconstruct a signal from values sampled at discrete, uniform intervals as long as the signal frequency is less than half the sampling frequency, fulfilling the Nyquist frequency criterion. Results of the simulation of sampling and reconstruction carried out with this developed model and a single data set is thus presented.

A sampling period value of 0.00001 was used and the data contained a single frequency component of 100 $\mathrm{KHz}$. Figure 3 shows the plot of the original pulse. Figure 4 shows the sampling of the pulse using the Fourier transform method. The sampling was done after every 10 data sets to obtain a total of 250 samples. Figure 5 shows the reconstruction of the pulse using the Fourier transform method. The shapes of the reconstructed and measured original pulse in agree quite well. Here, the magnitude of the reconstructed pulse can be seen to be slightly underestimated compared to that of the original measured pulse. The original measured pulse starts at a negative time while the reconstructed pulse starts from zero. This is because the reconstruction method does not take into consideration the negative time data values. The results of the simulation show that the developed mathematical model can carry out good event reconstruction.

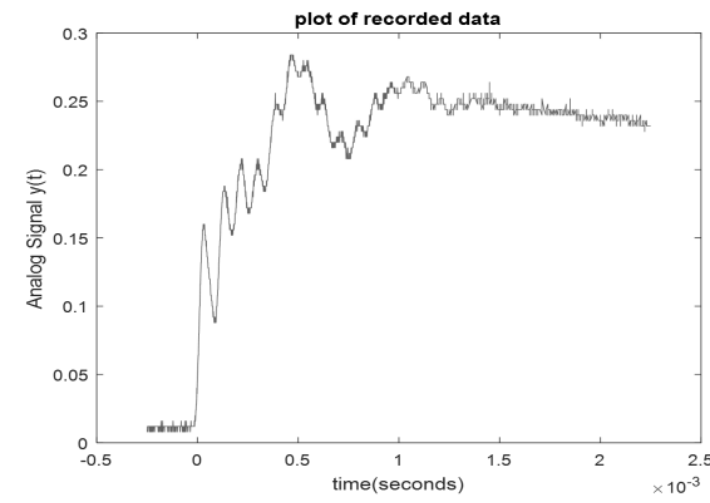

Figure 3: Plotted original pulse $y(t)$ of data set 1 in the time domain

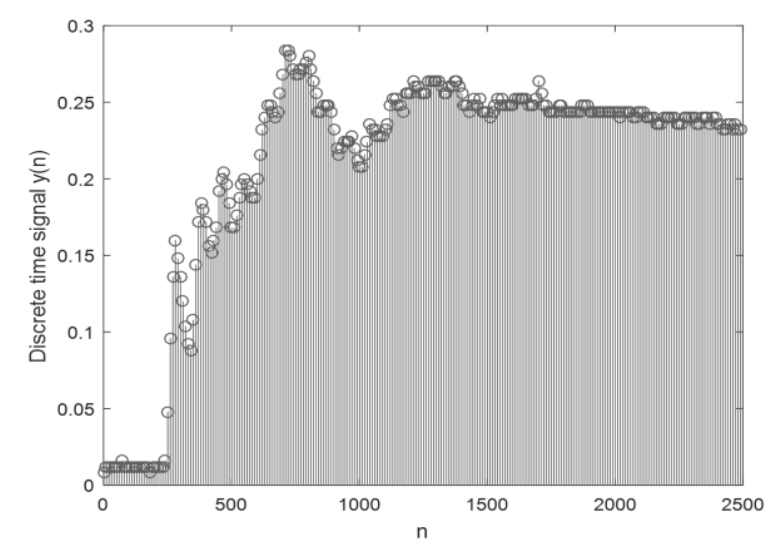

Figure 4: Sampled pulse $y(n)$ of data set 1 in the frequency domain

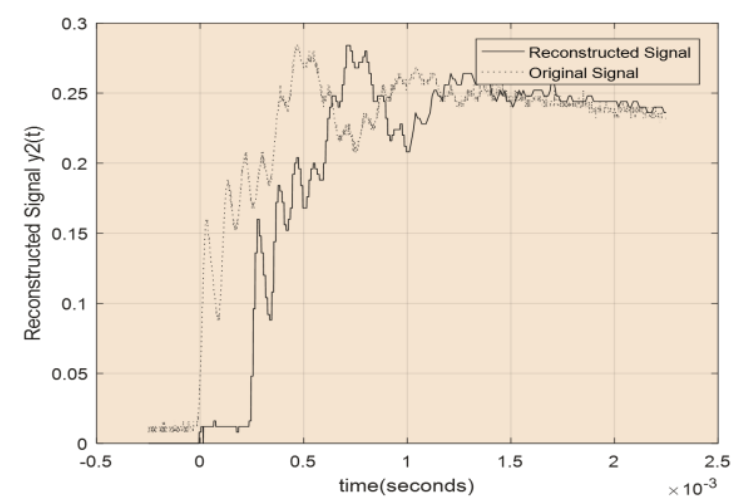

Figure 5: Reconstructed simulated pulse y2 (t) of data set 2 in the time domain (single frequency)

\subsection{Characterization of Damage}

\subsubsection{Event Reconstruction}

Figure 6 shows the Pico Log representation of the pressure pulses captured at each of the six sensors located along the pipeline. A sampling rate of $13.16 \mathrm{Ks} / \mathrm{s}$ was used in measuring and recording the pulse signals at the four sensors. The mathematical models for sampling in Equation 8 and the mathematical model for reconstruction in Equation 13 were applied through codes written in Matlab ${ }^{\circledR}$ to the data obtained from one of the many experiments 
made using the pulse generator and static air in the pipe as shown in Figure 5 . The six sensors were connected to six different channels on the data logger and the original pressure pulses captured at all sensors were labelled as s1, s2, s3, s4, s5, and s6 respectively, all located at various distances as discussed earlier. The tee connection was located between sensors 1 (s1) and 3 (s3). Sensor 2 (s2) was closest to the tee connection and was taken to be the actual event location.

Two major reconstructions of the pressure pulse at s2, the event location, were carried out. In the first reconstruction process, the original pressure pulse, s2 as in Figure 7 was sampled and the obtained samples used to reconstruct the event at $\mathrm{s} 2$. The original pulse s2 was made up of a total of 1320 samples and the sampling was carried out for 300 samples of s2. The sampling process converted the original pressure pulse $s 2$ to its discrete form. The original pressure pulse was then recovered from the discrete form of s2 as shown in Figure 8. The original pressure pulse was captured with a single sampling frequency of 13.16 $\mathrm{Ks} / \mathrm{s}$. A single sampling frequency of $26.32 \mathrm{Ks} / \mathrm{s}$ was used in order to achieve proper reconstruction of event, satisfying the Nyquist criterion.

Figure 8 shows a fairly good reconstruction of the actual event as the original pressure pulse ad the reconstructed pressure pulse have similar shapes. The amplitude peak of the reconstructed pressure pulse is a little bit underestimated compared to the original pressure pulse. This was consistent with all other reconstructions carried out on other data readings obtained from the several experimental results.

A second reconstruction of the actual event, s2 was carried out. In this case, an attempt was made to recover the original pressure pulse at the event location from the two closest pressure pulses at the locations closest tn the event location. This was done in other to provide a solution to real life situations where the signal from the sensor at the location of an event was lost or not captured at all. If the other sensors closest to the event captured the signals from the event, these signals can then be used to provide a clue as to what originally happened at the site of the event. In this case, sensors 1 and sensors 3 were closest to sensor 2 at the event location. Therefore, the pressure pulses s1 and s3 were sampled respectively. Both pressure pulses were made up of a total of 1320 samples and they were both sampled for 300 points each.

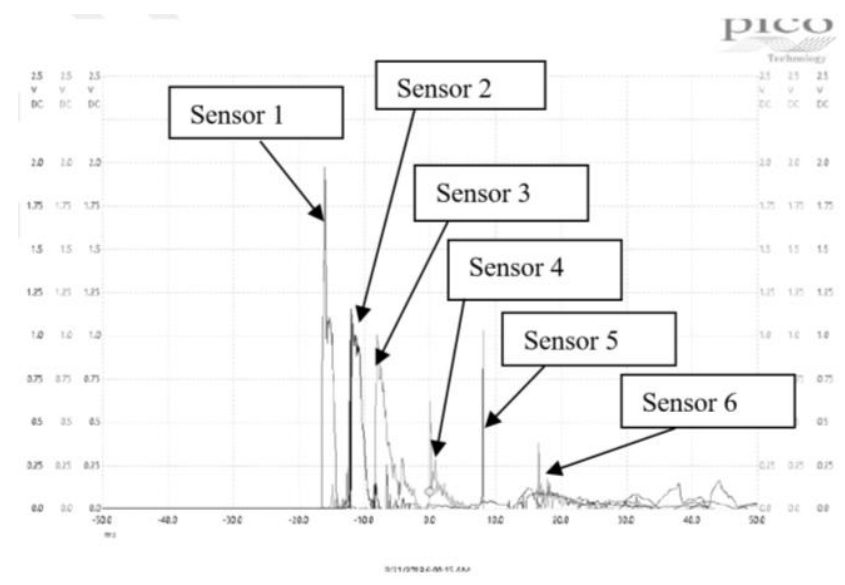

Figure 6: Pressure pulse measured at all six sensors of experimental rig at pressure of $1 \mathrm{bar}$

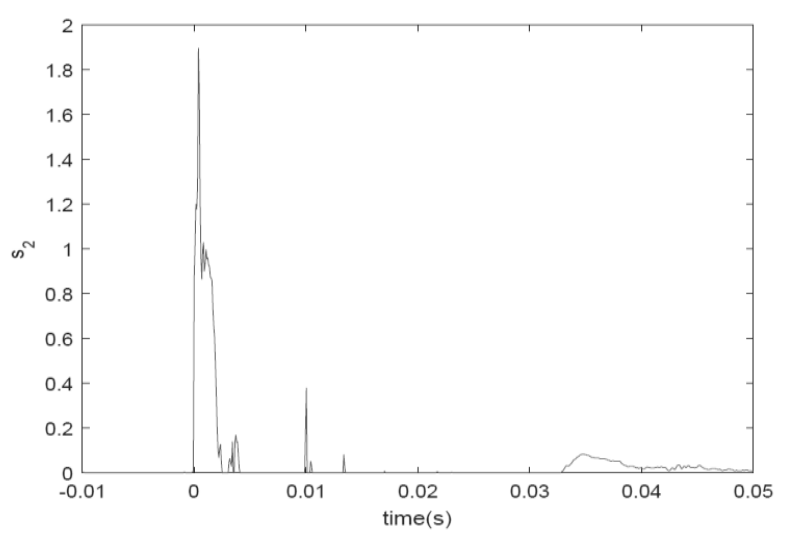

Figure 7: Matlab ${ }^{\circledR}$ plot of pressure pulse at sensor 2

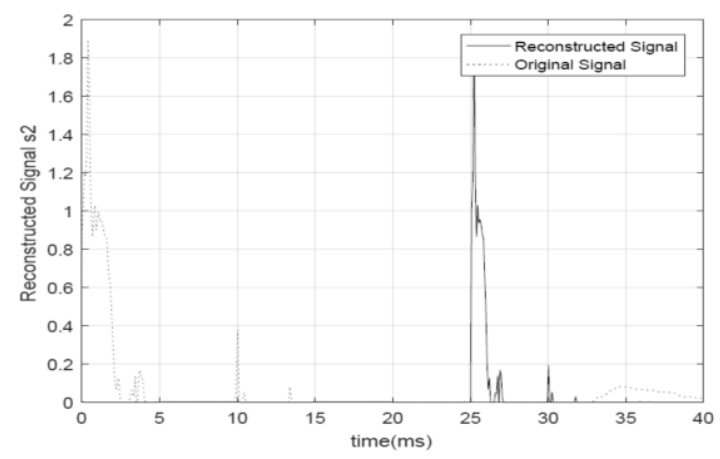

Figure 8: Reconstruction of original pressure pulse s2 from samples of $s 2$

The original pressure pulse s2 was then recovered from the combination of the discrete forms of both $\mathrm{s} 1$ and $\mathrm{s} 3$. Using the reconstruction model in Equation 13 in the Matlab ${ }^{\circledR}$ environment, a combination of these discrete forms gave a reconstruction of $\mathrm{s} 2$ as shown in Figure 9. A single sampling frequency of $26.32 \mathrm{Ks} / \mathrm{s}$ was used. This was double the value of the sampling frequency that was used in the experiment so as to satisfy the Nyquist criterion and ensure proper reconstruction. A good reconstruction of the actual 
event was achieved as the reconstructed original pressure pulse $s 2$ in Figure 9 had a similar shape with that of $s 2$ in Figure 7. As before, it was observed that they was a difference in the magnitude of $s 2$, as the reconstructed pulse was underestimated compared to that in Figure 7 showing the plot of $s 2$.

The magnitude of s2 in Figure 8 differed by $45 \%$ compared to its magnitude in Figure 7. This was consistent for all the repeated tests and the underestimation ranged between 40 to $45 \%$. To solve the problem of underestimation of the reconstructed pulse as had been observed with several experimental data processed, a reconstruction factor, $\mathrm{K}$ was introduced as see in Equation $14 . \mathrm{K}$ is an approximation factor obtained by dividing the maximum value or amplitudes of the original pressure pulse, $x_{o}(t)$ by the maximum value of the reconstructed pressure pulse, $x_{r}(t)$. Comparing Figure 9 and Figure 7, it is observed that the original pressure pulse in Figure 7 has amplitude of 1.9 while the reconstructed pulse in Figure 9 has amplitude of 0.85 . Therefore $\mathrm{K}$ is calculated as:

$$
K=\frac{1.9}{0.85}=2.235
$$

Using this obtained $\mathrm{K}$ value in Equation 14 in, Matlab $^{\circledR}$, a better reconstruction is obtained as shown in Figure 10. Figure 10 shows that a good reconstruction of an event is better achieved by applying a factor $K$ to the developed Fourier transform based model. This helped to solve the problem of underestimation of the nodes of the reconstructed signal that was observed with all the data samples that were processed.

\subsection{Damage Pattern Recognition}

One very important aspect of this work that was obtained from the experimental data is that a broad range of damages can be detected by this system. It was observed that each damage scenario generated its own pressure pulse that was peculiar to it when compared to the pressure pulse from another damage event. The four different experiments to simulate damage caused by explosion, drilling, vehicular motion and corrosion confirmed this.

\subsubsection{Damage from Explosion}

Figure 11 shows the typical pressure pulse that was obtained from the experimental test rig for tests to mimic damage caused by explosion. A pulse generator with a hand pump connected to it was used to generate sharp fronted pulses into the pipe. This was done repeatedly at pressure readings from 0.2 bar to
1 bar within the pulse generator. A total of 50 tests were taken and all the measured pressure pulses had a similar shape to that in Figure 14 . Only the amplitudes of the pulses differed due to the different pressure readings used.

\subsubsection{Damage from Drilling}

With the same experimental test rig, damage from a drilling event was mimicked. Two different hand drills were used to generate sharp fronted pulses into the pipe. The drills had power ratings 600 Watts and 800 Watts respectively. The drills were used to make holes along the pipe and the captured pressure pulse is shown.

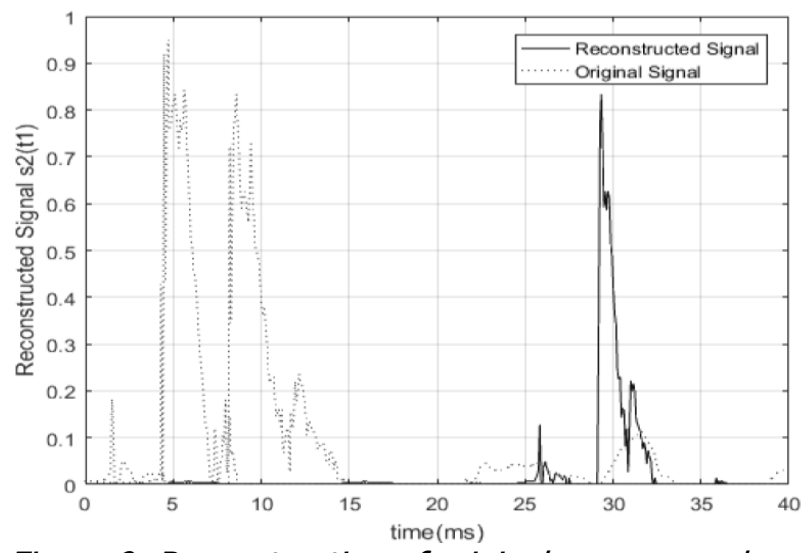

Figure 9: Reconstruction of original pressure pulse s2 from pressure pulses s2 and s3

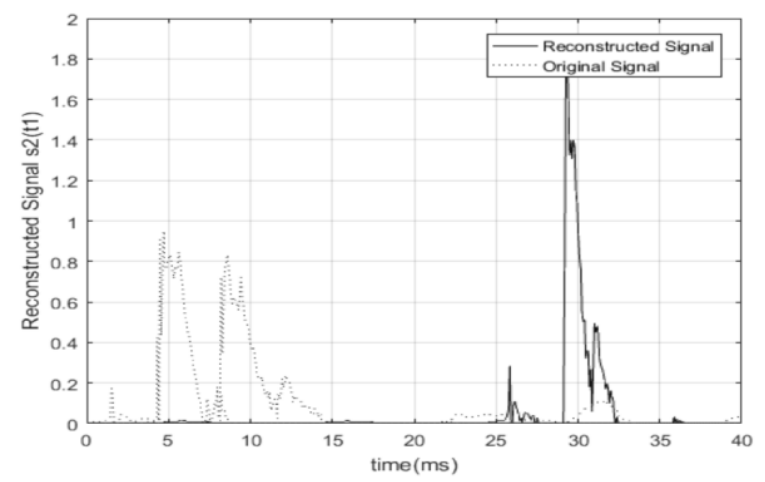

Figure 10: Reconstruction of original pressure pulse s2 from pressure pulses $s 2$ and s3 using reconstruction factor

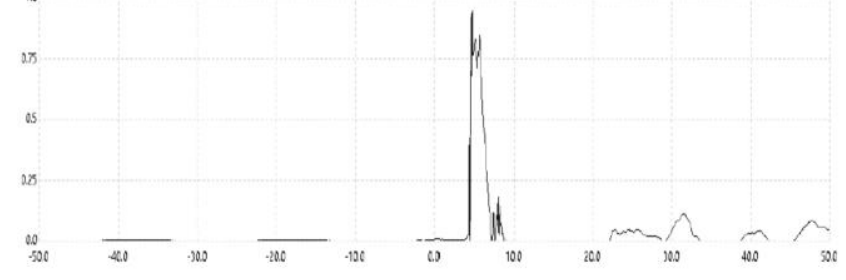

Figure 11: Typical pressure pulse measured at experimental rig to mimic damage due to explosion ( 0.8 bar pressure reading at single sensor) 
Figure 12 shows the typical pressure pulse for a test to mimic a drilling operation on a pipe. These pressure pulses were consistent for all the 30 repeated tests with 5 tests carried out at each sensor using either the 600 Watts or the 800 Watts input power hand drill. The results show that the pressure pulses from a drilling operation were different from that of an explosion as evident in the shape and amplitudes of the measured pressure pulses.

\subsubsection{Damage from Vehicular Motion}

To mimic potential damage to a pipe caused by movement of the tires of a vehicle over the pipe, a hammer was used to impart a mass on the pipe of the experimental test rig thereby introducing sharp fronted pulses into the pipe.

Figure 13 shows the typical pressure pulse for a test to mimic the movement of a vehicle over a pipe. The measured pressure pulses were consistent for all the 30 repeated tests with 5 tests carried out at each sensor using a small wooden-handle hammer. The results show that the pressure pulses from a heavy mass as that obtainable from the mass of a vehicles' tyres are different from that of an explosion or drilling operation as evident in the shape and amplitudes of the measured pressure pulses. It was observed that these measured pulses were a little bit similar to those of an explosive event but the difference was that the pulses from the hammer experiments were smoother that those gotten from the explosion tests.

\subsubsection{Damage from Corrosion}

To mimic damage from corrosion, a corrosive environment was created within the pipe. The pipe was connected to a water tank and water was allowed to flow through the pipe. A little hole was drilled at positions close to each sensor location and a pipette was used to introduce acid in varying quantities into the water filled pipe. At first, $2 \mathrm{ml}$ of acid was introduced into the pipe; then $5 \mathrm{ml}, 10 \mathrm{ml}$ and $25 \mathrm{ml}$ respectively. $75 \%$ and $100 \%$ concentrated $\mathrm{HCL}$ respectively was used and it was intended that the introduction of the acid into the water would create an environment corrosive enough to generate sharp fronted pulses within the pipe. Substantial pressure pulses were noticed only when $10 \mathrm{ml}$ and $25 \mathrm{ml}$ of the $100 \%$ concentrated acid were introduced, with no pulses observed when $75 \%$ concentrated acid was used for both $10 \mathrm{ml}$ and $25 \mathrm{ml}$ volumes. This could have been as a result of the increase in the resistivity of the piezoelectric sensors after it came in contact with water. Also repeated tests could not be carried out because the piezoelectric sensors lost their conductivity after sensing as they came in contact with the acid.

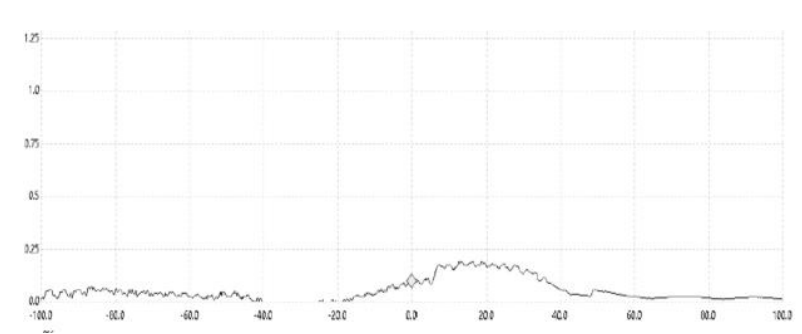

Figure 12: Typical pressure pulse measured at experimental rig to mimic damage due to drilling (800 Watts drill input power at single sensor)

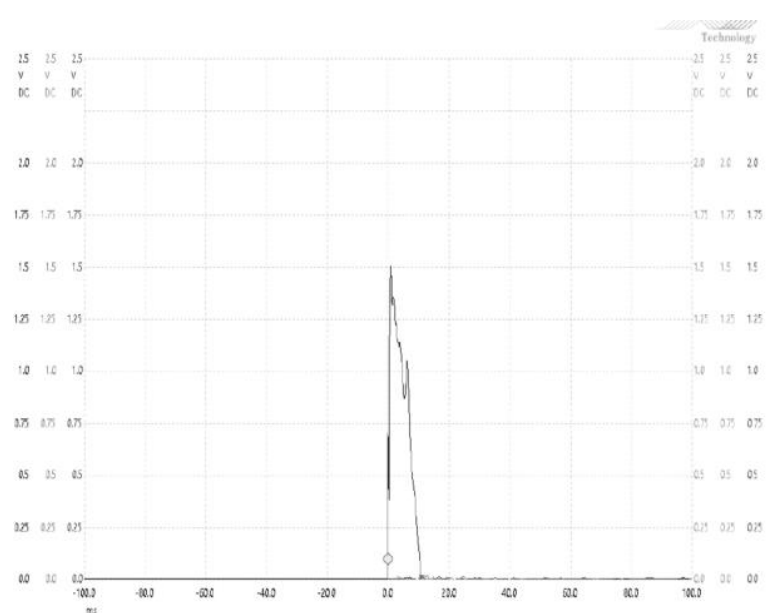

Figure 13: Typical pressure pulse measured at experimental rig to mimic damage due to vehicular motion (measurement at sensor 3)

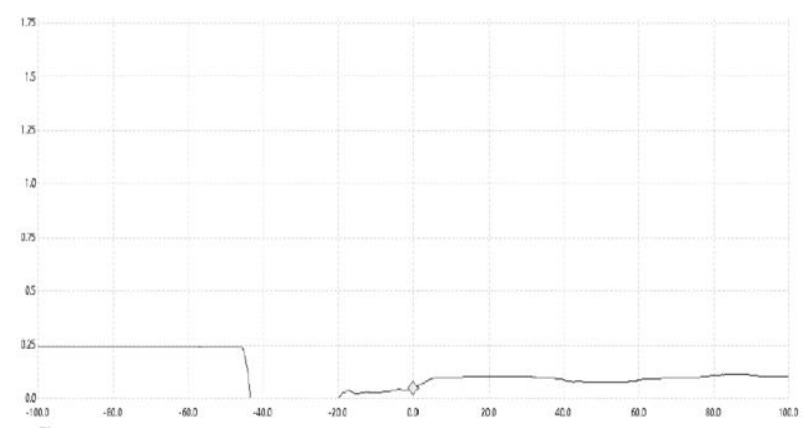

Figure 14: Typical pressure pulse measured at experimental rig to mimic damage due to acid $(25 \mathrm{ml}$ of acid)

Figure 14 shows the typical pressure pulse from an experiment to mimic damage due to corrosion. The results obtained when $10 \mathrm{ml}$ of acid was used showed very little pulse while a more visible pulse was observed when $25 \mathrm{ml}$ acid was used. The pattern in both cases of acid quantity was not consistent to develop a pattern, but the results were different from those observed in the other damage scenarios. 


\section{CONCLUSIONS}

Impacts and intrusions are among the major causes of pipeline damage. These events cause vibrations in the source point that can be used to characterize the defect. In this work, various mathematical techniques; algorithms and numerical experiments for real time monitoring of pipelines have been presented, developed and discussed. This system was tested and the main results are as follows:

1. Fourier transform based algorithms can be used to characterize and classify damage events on a pipeline through the process of event reconstruction.

2. The problem of underestimation of the reconstructed pulse can be solved through the use of an underestimation factor $\mathrm{K}$ in the reconstruction model.

3. The reconstruction of the measured original pulse at a damage location can be achieved from combining the measured pulses from two other close locations using the developed Fourier transform based model.

4. This model can be applied to real pipelines to classify and characterize damage events. This is seen from the experiments carried out to mimic different damage scenarios-damage due to explosion, drilling, vehicular movement and corrosion respectively. It is observed clearly from the results obtained that each damage scenario that leads to leakage has its peculiar pressure pulse pattern. This is because these damage scenarios occur at different pressure ratings thereby causing different vibrational patterns.

\section{ACKNOWLEDGMENT}

This research did not receive any specific grant from funding agencies in the public, commercial, or not-forprofit sectors.

\section{REFERENCES}

[1] Agrawal, A. "A theoretical, numerical and experimental investigation of guided wave propagation in hollow cylinders" www.researchgate.net/.../276129611, Accessed on May 10, 2016.

[2] Park, H. W., Sohn, H., Law, K .H., and Farrar, C. R. "Time reversal active sensing for health monitoring of a composite plate", Journal of Sound and Vibration. Vol. 302, No. 1-2, 2007, pp 50-66.

[3] Razi, P.; Esmaeel, R.A., and Taheri, F. "Improvement of a Vibration-based Damage Detection Approach for Health Monitoring of Bolted Flange Joints in Pipelines. Struct. Health Monit. Vol. 12, 2013, pp 207-224.

[4] Olugboji, O. A. "Development of an impact monitoring system for petroleum pipelines", www.journal.au.edu/au techno/2011/oct2011/j ournal152 article08.pdf, Accessed May 12, 2106.

[5] Junxiao, Z., Liang, R., Siu-Chun, H., Ziguang, J., Gangbing, S. "Gas pipeline leakage detection based on PZT sensors", Smart Materials and Structures, doi:10.1088/1361-665X/26/ 2/025022, Accessed June 20, 2018.

[6] Guofeng, D., Qingzhao, K., Hua, Z., Haichang, G. "Multiple cracks detection in pipeline using damage index matrix based on piezoceramic transducer-enabled stress wave propagation", Sensors, doi:10.3390/s17081812, Accessed June 20, 2018.

[7] Golmohamadi, M. "Pipeline leak detection", http://scholarsmine.mst.edu/masters theses/73 97, Accessed on April 11, 2016.

[8] Changhang, X., Jing, X., Wuyang, Z., Qingzhao K., Guoming, C., Gangbing, S. "Experimental investigation on the detection of multiple surface cracks using Vibrothermography with a lowpower piezoceramic actuator. Sensors, doi:10.3390/s17122705, Accessed June 20, 2018.

[9] Enrique, S., Rui, S., Ricardo, P. "Damage detection based on power dissipation measured with PZT sensors through the combination of electromechanical impedances and guided waves", Sensors, doi:10.3390/s16050639, Accessed July 23, 2018.

[10] Kia, X., Qingshan, D., Lujun, C., Siuchun, H., Gangbing, S. "Damage Detection of a concrete column subject to blast loads using embedded piezoceramic transducers", Sensors, doi:10.3390/s18051377, Accessed July 25, 2018.

[11] Blackledge J.M. Digital Signal Processing, Dublin Institute of Technology, School of Electrical and Electronic Engineering. 2006.

[12] Goswami, J. C., Chan, A. K. "Fundamentals of Wavelets: Theory, Algorithms, and Applications", New York, Wiley. ISBN 0-471-19748-3. 